\title{
NUEVA APORTACION AL CONOCIMIENTO DE LOS PAVIMENTOS MUSIVOS EN LA VILLA ROMANA DE EL RUEDO (ALMEDINILLA, CORDOBA)
}

\author{
Manuel Fdo. MORENO GONZALEZ *
}

\section{Resumen}

Pretendemos en el presente artículo dar a conocer los esquemas ideales, obtenidos mediante tratamiento informático, de cinco de los mosaicos romanos de la villa de El Ruedo (Almedinilla, Córdoba), contribuyendo así a completar el estudio monográfico que hace ya algunos años fue publicado en esta misma revista (HIDALGO, 1991), e incorporando las numerosas novedades que se vienen produciendo en la investigación sobre el yacimiento (VAQUERIZO, 1990; VAQUERIZO et alii, 1994; VAQUERIZO, CARRILLO, 1994, e.p.; CARRILLO, 1990 y 1992) (1).

\section{Summary}

In the present article we aim to report the ideal reconstructions, obtained by computer treatment, of five Roman mosaics from the villa of El Ruedo (Almedinilla, Córdoba), contributing in this way to complete the monograph published in this same magazine some years ago (HIDALGO, 1991), and incorporating the latest details from the continuing investigation of the site.

Entre las vías de estudio e investigación que se han abierto últimamente en relación al yacimiento de El Ruedo destaca el estudio arquitectónico del mismo mediante tratamiento informático, proyecto en el que trabaja J. L.Vaquerizo, con el objetivo de obtener el levantamiento planimétrico de la villa, así como la reconstrucción ideal de las decoraciones parietales y musivas. De él han derivado las cinco reconstrucciones ideales de mosaicos que ahora presentamos como primer avance.

(*) Universidad de Cordoba.

(1) Este artfculo obedece al requerimiento que, en aras al conocimiento progresivo de los trabajos que, sin interrupción, se vienen realizando en la villa romana de El Ruedo, nos hizo en su momento el Prof. Dr. Desiderio Vaquerizo, a quien agradecemos su apoyo y estímulos constantes. Del mismo modo, gracias a J. L.Vaquerizo por el tratamiento informático de los mosaicos y su colaboración. Finalmente, a todas aquellas personas que han facilitado nuestra labor, de manera muy particular a la Profra. Dra. Pilar León, quien nos ha abierto las puertas a la investigación, y a todos los compañeros del Seminario de Arqueología de la Universidad de Córdoba. 
Del mismo modo, creemos interesante nuestro aporte por integrar los mosaicos en el ambiente exacto para el que fueron concebidos, algo que ha sido posible merced a la interpretación funcional del yacimiento realizada por J. R. Carrillo con motivo de su Tesis Doctoral (CARRILLO, 1992), de la que recientemente hemos conocido un primer avance (VAQUERIZO et alii, 1994). Este autor acomete el análisis arquitectónico de la villa, buscando la interpretación funcional de las estancias y su inserción en el conjunto edilicio de aquélla, utilizando como items básicos los arquitectónicos: planta, dimensiones y tipo de decoración.

Carrillo, basándose en las refectiones arquitectónicas que se advierten en la villa de El Ruedo, llega a establecer cuatro fases constructivas (CARRILLO, 1992, 52-69) que abarcarían desde mediados del siglo I hasta un momento posterior a mediados del siglo V. Los mosaicos que aquí presentamos pertenecen a la Fase III que, cronológicamente, corresponde al período que va desde finales del siglo III-inicios del siglo IV hasta mediados del siglo $\mathrm{V}$, siendo ésta la etapa en la que se realizan las renovaciones arquitectónicas que más enriquecen estéticamente a la zona residencial de la villa.

Sintetizando muchísimo, la villa de El Ruedo responde a un esquema muy extendido en Andalucía (FERNANDEZ CASTRO, 1978, 326) en el que la vida se desarrolla en torno a un peristilo (VAQUERIZO et alii, 1994, 67 ss) -habrá que esperar las conclusiones del estudio informático de la villa para confirmar si contó además con un atrio, al que habría servido de impluvium la cisterna que se localiza en el sector sur de la misma, por donde se realizaría la entrada (D. Vaquerizo. Com. personal)-; cuenta con estancias de representación y prestigio como triclinium y exedra, situada en eje con un posible oecus absidado, y fue dotada de una sistemática decoración musivaria, cuya datación cronológica parece coincidir con las grandes refectiones que, como sucede en otras villas de Andalucía (JIMENEZ SALVADOR, MARTIN-BUENO, 1992, 71 ss.), se llevan a finales del siglo II, una, y a finales del siglo III-principios del siglo IV, la otra.

En la villa de El Ruedo conocemos de hecho pavimentos musivos remontables ya a aquel primer momento. Sin embargo, el más importante conjunto musivario se encuadra en la última etapa, aunque salvo en el caso de los cinco que ahora reestudiamos, nos han llegado muy fragmentados, pese a lo cual podrán seguramente ser reconstruidos. Mientras tanto, aportamos ahora los esquemas ideales de los cinco mejor conservados con la idea de proporcionar a la investigación una documentación fiable sobre ellos. Ahora bien, no pretendemos, de ningún modo, enmendar aportación alguna de las ya realizadas por el primer investigador que los ha tratado (HIDALGO, 1991), sino tan sólo matizar o como mucho ampliar algunas de sus apreciaciones, contribuyendo en definitiva al mejor conocimiento de los pavimentos musivos, siempre en opus tesellatum, de esta villa cordobesa.

En los últimos años los estudios sobre mosaicos en Hispania se caracterizan por la dispersión. Destacan algunas obras de carácter recopilatorio como la de J. M. Blázquez (BLAZQUEZ, 1993), con la que viene a completar sus Corpora musivos, aún 
en curso, $\mathrm{o}$ algunas otras dedicadas a períodos concretos, como los siglos altoimperiales (DURAN, 1993) o la Antiguiedad Tardía (GUARDIA, 1992), en las que se acometen importantes esfuerzos de síntesis e interpretación iconográfica. Ni siquiera ocurre así con las publicaciones dedicadas al mosaico de tema geométrico, entre las que continúan en vigor las clásicas de Ovadiah (OVADIAH, 1980), Balmelle (BALMELLE et alii, 1985) o Fernández-Galiano (FERNANDEZ-GALIANO, 1980), obras en las que se basan incluso los más recientes estudios, caso del Dicionário de motivos geométricos no mosaico romano (VIEGAS, C., ABRA OS, F., MACEDO, M., 1993), al que por su reciente aparición aún no hemos tenido acceso.

En Hispania la dispersión es, pues, característica importante. Aún así, contamos con algunos simposia de enorme interés (AAVV, 1989; AAVV, 1990) (2); obras dedicadas a regiones (RAMALLO, 1985; FERNANDEZ-GALIANO, 1987) o problemáticas concretas (ARCE, 1993; GOMEZ, 1992 y 1993); yacimientos o áreas arqueológicas específicas (FERNANDEZ-GALIANO, 1989; JARREGA, 1993) o a los mosaicos en su relación con los asentamientos de tipo rural (FERNANDEZ CASTRO, 1978 y 1982). Tal circunstancia se agrava para el caso de la Bética, donde son pocas las obras recientemente publicadas y casi todas para el caso de Málaga (RODRIGUEZ OLIVA, 1987). Para Córdoba, salvo los estudios monográficos sobre la Casa del Mitra, en Cabra (BLANCO, GARCIA, BENDALA, 1972; IIMENEZ, MARTIN-BUENO, 1992), y la villa de Fuente Alamo en Puente Genil (DAVIAULT, LANCHA, LOPEZ PALOMO, 1987), así como alguna revisión iconográfica sobre mosaicos de especial interés (LOPEZ MONTEAGUDO, 1991), el panorama es desalentador, lo que no implica, en absoluto, ausencia de hallazgos, sobre todo si hemos de juzgar por los resultados que da el vaciado del Anuario Arqueológico de Andalucía, en sus volúmenes correspondientes a los años 1985-1990.

De aquí la necesidad, y la urgencia, de acometer un estudio en profundidad que tienda a la sistematización y catalogación de los pavimentos marmóreos y de opus tesellatum en la provincia Baetica, atendiendo a la funcionalidad de los espacios en que se insertan y a sus valores simbólicos, ideológicos e iconográficos, de acuerdo a los más recientes aportes de la investigación. Es esta la razón de que hayamos emprendido una nueva línea de trabajo que sobre el tema: Pavimentos musivos y marmóreos en edificios públicos y privados de Baetica: aspectos técnicos, funcionales, estilísticos e iconográficos, se inserta en el macro- proyecto de recreación arqueológica de la

(2) Entre las últimas iniciativas en relación al estudio del mosaico, merece una mención especial el curso recientemente realizado bajo la dirección de los Profres. Drs. Mercé Roca y Josep Ma Gurt, de la Universidad de Barcelona, en el que han participado la Profra. Dra. Catherine Balmelle (CNRS, París), la Profra. Dra. Aisha Ben Abed (Museo del Bardo, Túnez) y el Prof. Dr. Jean Pierre Darmon (CNRS, París), bajo el título Mosaics de l'arquitectura civil i religiosa a l'antiguitat tardana. La producció i la decoració musives de l'arquitectura domestica i dels edificis religiosos a Orient i a Occident. Implicacions socials $i$ culturals. Agradecemos desde aquí a la Profra. Dra. Mercé Roca el habernos facilitado la documentación de un curso cuyo lítulo refleja las nuevas corrientes en el estudio del mosaico romano. 
Colonia Patricia Corduba, dirigido por la Profra. Dra. Pilar León, a quien desde aquí agradecemos su apoyo y estímulo constantes.

El hecho de que no hayamos podido estudiar de visu los mosaicos de El Ruedo -algunos, trasladados a los almacenes Municipales del Ayuntamiento de Almedinilla; otros, conservados in situ bajo una capa de escombros que los excavadores dispusieron cuando aún no se conocía el destino de la villa- dificulta algunos aspectos de su estudio, entre los cuales podemos destacar:

- El porcentaje de teselas por decímetro cuadrado. Es ésta una información de vital importancia para determinar la dificultad del mosaico, la calidad del diseño y del artista, el mayor o menor coste del trabajo, así como el tipo de opus utilizado.

- El material en el que éstas se han realizado, pues aunque normalmente se utilizan piedras locales, a veces son de terracota, mármol o pasta vítrea o incluso de materiales traídos de otros lugares.

- La estructura sobre la que se asientan los pavimentos. La urgencia que condicionó todos los trabajos arqueológicos en la villa impidió en su momento la realización de sondeos bajo la cama de los mosaicos, algo que después no ha podido tampoco realizarse por dificultades administrativas que no vienen al caso.

Por tanto, en el estudio que nos ocupa quedan aún aspectos abiertos a la investigación. Entre ellos, nos parece de primordial interés el relacionado con la participación del propietario en la elección de los motivos representados en los mosaicos, pues si para el caso de la escultura parece quedar clara la elección del programa iconográfico (VAQUERIZO, 1990 b; VAQUERIZO et alii, 1994, 83 y ss.; JIMENEZ SALVADOR, MARTIN-BUENO, 1992, 59 y ss.), debemos suponer algo similar para la ornamentación de carácter musivo. Es este un tema en continua discusión; así, algunos investigadores que en algún momento subestiman el papel jugado por el cliente a la hora de hacer la elección "por desinterés, incompetencia o imposibilidad" (THEBERT, 1988, 339), no tienen inconveniento más adelante -creemos que de manera mucho más acertada, a juzgar por lo que ocurre en yacimientos como el que nos ocupa- en concluir que al menos quien hace el encargo juega un papel esencial, al decidir "en función de sus necesidades y de consideraciones financieras" (THEBERT, 1988, 341).

Del mismo modo, se continúa sin llegar a un acuerdo en lo que se refiere al tema de la mayor o menor influencia africana, en mosaicos de esquema geométrico principalmente, de la Bética y particularmente de los que ahora nos ocupan.

A este respecto, sabemos que existieron relaciones comerciales entre ambas zonas del Imperio, que en el caso de El Ruedo se constatan además por la presencia de abundante cerámica africana de importación (SIERRA FERNANDEZ, 1994, en este mismo volúmen). Lógicamente, cuanto más al interior menor suele ser el porcentaje en la aparición de elementos de importación como la cerámica, afirmación que se constata si comparamos, siguiendo al mencionado investigador, El Ruedo con yacimientos costeros como Cártama, donde los porcentajes son bastante superiores. Es 
significativa, además, la coincidencia cronológica que existe entre los mayores conjuntos de material cerámico de importación africana del siglo IV localizados y la datación de los mosaicos que aquí presentamos.

Cuestión aparte es, sin embargo, la del orígen geográfico de los talleres o artistas ambulantes que realizaron por ejemplo los mosaicos de El Ruedo; hoy pensamos en Córdoba por pertenencia administrativa; no obstante, pudieron llegar a El Ruedo utilizando esos mismos canales comerciales que debieron nutrirse no sólo de cerámicas, animales o productos exóticos como el marfíl, sino también de emblemata musivos, "cartones" y quién sabe si de los propios artesanos.

$$
* * *
$$

Pasemos ahora al estudio de estos cinco pavimentos musivos, para el que nos basaremos en las descripciones que sobre ellos se han hecho (HIDALGO, 1991 y 1994) y en la Tesis Doctoral de Carrillo (CARRILLO, 1992). Lo hacemos sin considerar determinante la numeración aplicada con anterioridad a los mosaicos (HIDALGO, 1991 y 1994), para exponerlos estancia por estancia y de norte a sur, primero en el ala oeste y por último en el ala este de la casa.

Estancia LIX: (3) (Fig. 1)

Se accede a ella desde la Estancia LXI, y es identificada por Carrillo (CARRILLO, 1992, 296) como dormitorio, de acuerdo con la opinión previa de Thebert que entiende este tipo de estancias yuxtapuestas, en las inmediaciones del peristilo, como un binomio entre salas de recepción y cubicula, esquema muy repetido en el Africa romana (THEBERT, 1988, 349-350). Existen planteamientos distintos en relación a la funcionalidad de estos espacios que los relacionan más bien con salas de aparato en invierno o comedores, a los que se accede desde una antecámara ( $c f r$. ENNABLI y BEN OSMAN, 1983, 133). No obstante, en el caso de El Ruedo, donde ya existen espacios de representación (estancias XVII, XXXVII y probablemente XXXII), tal pluralidad sería seguramente innecesaria (CARRILLO, 1992, 296).

El mosaico que se inserta en esta estancia (HIDALGO, 1991, N² 2, 330 ss., Lám. IV; 1994, $\mathrm{N}^{\circ} 2$, 16, fig. 3), sea comedor privado o espacio para el descanso es, al igual que el resto de los pavimentos musivos que nos han llegado de El Ruedo, de composición decorativa geométrica, en la que domina el trazo recto y una variada policromía, reservando el color negro para los elementos de separación.

Domina el campo compositivo una estrella formada por ocho losanges. En los ángulos se disponen sendos cuadrados, en los que se insertan nudos de Salomón rodeados de otro cuadrado al que enmarcan dientes de sierra escalonados. Por su parte,

(3) El esquerra de este pavimento, así como el de la estancia XXXVII, han sido recogidos en una publicación reciente (VAQUERIZO et alii, 1994, 81-82, fig. 35-36), donde aún se observan algunos errores en sus motivos decorativos, hecho derivado del propio tratamiento informático, sin finalizar todavía en aquel momento; dichos errores aparecen subsanados en las láminas que aquí presentamos. 
los triángulos que forman la unión de las losanges en cada lado, delimitados por un denticulado negro, contienen una pelta con medias cruces en sus vanos.

Todo el campo decorativo anteriormente descrito es encuadrado con una trenza de tres cabos polícroma.

Los paralelos de la estrella de ocho losanges son muchos y se extienden a lo largo de todo el Imperio Romano, con una cronología muy amplia, por todo lo cual es difícil utilizarlo como criterio de datación (SALIERS, 1974).

\section{Estancia LXI: (Fig. 2)}

Habitación de carácter privado (CARRILLO, 1992, 296). Debió servir como centro de distribución, a manera de estancia secundaria, preservando de la inmediata proximidad de un espacio de paso como es el peristilo, a tres estancias contiguas a las que da acceso: LIX, LX y LXII, identificables con lo que Plinio el Joven denomina cubiculus noctis et somnii (II, 17, 22). A la segunda de ellas se accedería mediante escaleras, de construcción previa a la composición del mosaico, que se diseña respetándola. Uno de los motivos que hacen pensar a Carrillo que se trata del distribuidor de una serie de alcobas es que el vano de entrada aparece desplazado en relación al de las otras estancias, seguramente con el fin de resguardarlas de la luz directa, que de no ser así entraría sin matices desde el peristilo ( $c f r$. PACKER, 1967: 136 n $^{\circ} 31$ ).

Pues bien, el pavimento de este distribuidor aparece decorado por un mosaico con ruedas de peltas no contiguas que enmarcan sendos nudos de Salomón (BALMELLE, 1985, 348, pl.223,e.; HIDALGO,1991, Nº 1, 326 ss., láms. I-III; 1994, Nº 1, 16, fig. 2), realizado sobre un fondo blanco y que utiliza el negro como color dominante en la figuración geométrica, reservando la policromía sólo para algunos elementos decorativos. Es una composición totalmente geométrica basada en 33 ruedas de peltas rematadas en volutas, entre las que se sitúan pequeñas flores cuatripétalas. Toda ella aparece enmarcada por tres franjas: un filete triple con teselas negras, otro formando denticulado en negro y un tercero que es doble. Enmarca su lado N. una arquería formada por 7 peltas, con triángulo dentado en el apex (BALMELLE, 1985, 152, pl.97, c.) y con columnas en las que se representan capitel y basa. Desde el peristilo da paso a través de un cuadrado tangente cóncavo, polícromo, formado por un filete triple.

Además de los paralelos indicados por Hidalgo (HIDALGO, 1991), podemos señalar como simples ejemplos que la esvástica-pelta -un motivo bien conocido desde mediados del siglo II-, aparece inserta del mismo modo en un cuadrado rematado por cenefa triple de un mosaico de Chedworth y en otro aparecido en Woodchester, ambos en Britannia y datados en el siglo IV (SMITH, 1963), y enmarcando uno de los dos cuadrados que configuran el mosaico de la villa de Stonesfield (Oxfordshire), también en Britannia con la misma cronología que los anteriormente citados (DE LA BEDOJERE, 1993).

En Hispania, encontramos este motivo en la villa de Liédana (Navarra) (BLAZQUEZ, J. M.; MEZQUIRIZ, M. A., 1985), datado a fines del siglo II o principios del 
siglo III. También aparece en el mosaico número 3 de la villa de Pla de l'Horta (Gerona) (NOLLA,J. M.; SAGRERA,J., 1993), con la misma cronología que el anterior; en él, realizado exclusivamente en blanco y negro, la rueda de pelta que gira en torno a un nudo de Salomón, ocupa todo el campo del pavimento, repitiéndose 80 veces. Se le asigna una cronología que cabalga entre finales del siglo II y principios del siglo III, y constituye el ejemplo con mayor similitud al mosaico de la estancia LXI de El Ruedo.

Para el motivo de arquería corrida, contamos con un nuevo paralelo en la misma Colonia Patricia. Nos referimos a uno de los mosaicos exhumados en la campaña de excavaciones practicadas durante el verano de 1993 en el Paseo de la Victoria de Córdoba, donde este motivo aparece enmarcando un campo con decoración geométrica, a base de cuadrados superpuestos que forman estrellas de ocho puntas, y vegetal, con hojas de yedra y roleos, datado en el Informe de Excavación en el siglo IV (Com. personal, J. F. MURILLO).

En síntesis, creemos fuera de toda duda que la cronología de estos mosaicos de E1 Ruedo deba ser remontada a finales del siglo III o comienzos del siglo IV d. C. Es cierto que algunos motivos como el de las ruedas de peltas enmarcando nudos de Salomón podrían retrasarse casi en un siglo; no obstante, en este caso concreto contamos con pruebas de otro tipo suficientes para bajar su cronología hasta hacerla coincidir con la gran última refectio detectada en la villa: nos referimos al diseño del propio pavimento respetando una escalera que se construye para facilitar el acceso a la estancia LXII en el momento en que se la transforma en hipocaustum. Pensamos, por consiguiente, que nos encontramos ante una perduración de determinados motivos, bien por simple cuestión de preferencia estética, bien por un cierto mantenimiento en las modas o simplemente en la oferta de que disponían los talleres.

Estancia XXXVII: (HIDALGO, 1991, № 4, 335 ss.; láms. VII-VIII) (Fig. 3)

Pertenece esta estancia a una exedra de recepción, según J.R.Carrillo (CARRILLO, 1992, 281; VAQUERIZO et alii, 1994, 110), quien para llegar a esta conclusión se basa en distintos aspectos que caracterizan a este tipo de espacios, presentes, según Thebert (THEBERT, 1988, 362) en casas de cierta importancia: acceso directo desde el peristilo, mayor tamaño que el resto de las estancias y decoración mucho más cuidada, que incluye la única muestra de pintura parietal figurada que nos ha llegado en El Ruedo; además, las exedras suelen disponer de un único vano de entrada, bastante ancho (Cfr..MORERE, 1989, 23), y se suelen situar en eje perpendicular al triclinium ( $C f r$ REBUFFAT, 1969), siendo consideradas como un espacio dedicado a actividades culturales, utilizadas para la conversación y la lectura (FERNANDEZ CASTRO, 1982, 202), como una especie de despacho del propietario de la villa.

Fuera la realidad cual fuere, se eligió para esta estancia un mosaico con esquema compositivo a compás, en el que se desarrolla un repertorio geométrico y vegetal conformado de la siguiente manera: la composición aparece enmarcada por una banda ancha de teselas blancas en la que se inscribe una trenza triple polícroma (BALMELLE, 
1985, 122, pl. 72, d) que rodea el campo interior, formado por un octógono de lados curvos enlazados por otra trenza de dos cabos polícromos (BALMELLE, 1985, 120, pl. 70), que al inscribirse en un cuadrado forma ocho cuartos de círculo en los que aparece decoración vegetal. El interior del octógono está decorado con un círculo central rodeado por un cordón en $\mathrm{S}$ adyacentes en oposición de colores (BALMELLE, 1985, 117, pl. 67,c.). Se inscribe en él un motivo de cortina polícroma (BALMELLE, 1985, 155 , pl. 100,c.) dentada que rodea un tercer círculo que ocupa una flor de ocho pétalos, cuatro lanceolados alternando con otros terminados en volutas.

Los triángulos de los vértices aparecen rodeados por una franja polícroma de meandros compartimentados por fracciones imbricadas, en oposición de colores (BALMELLE, 1985, 75, pl. 32, d.), y en su interior se representan dos medias hojas lanceoladas y una central rematada en volutas.

Los cuartos de círculo alternan las siguientes decoraciones:

- Hoja de yedra con decoración de roleos vegetales a derecha e izquierda.

- Tres hojas de laurel flanqueadas a derecha e izquierda por roleos vegetales.

Es un modelo compositivo "a compás" (FERNANDEZ GALIANO, 1980), al que se asigna un origen arquitectónico, con una cronología a partir de Adriano y un origen geográfico en Italia, de donde se difundiría a la mitad occidental del Imperio; sin embargo, aunque este esquema tenga una difusión eminentemente occidental, también aparece en algún lugar del Mediterráneo oriental, como Hagia Tríada (WAYWELL, 1979), con una cronología establecida en el siglo II d.C..

El motivo de cortina polícroma aparece, además de en los casos señalados por Hidalgo (HIDALGO, 1991, 336-7), formando un esquema múltiple en el que, al igual que en El Ruedo, se inscribe en un octógono de lados curvos enmarcados por una trenza doble, en un mosaico de la Casa del Atrio Tetrástilo de Nora (Cerdeña), (ANGIOLILLO, 1987, 187, Fig. 99). Estas frecuentes semejanzas entre los mosaicos sardos y los hispanos se entienden si tenemos en cuenta las rutas comerciales marítimas que pasaban por el Estrecho de Bonifacio o por el sur de Cerdeña, transportando aceite hispano, garum, vino de la Tarraconense y minerales a Roma, y que volvían de regreso con mercancías tales como, por ejemplo, sarcófagos, con lo cual las relaciones hispanosardas debían ser importantes, a juzgar además por los hallazgos submarinos (BLAZQUEZ, 1993, 93 y ss.).

Un ejemplo de hoja de yedra con decoración de roleos a derecha e izquierda, datado en el siglo II, se encuentra en un mosaico bícromo hallado en la calle Hermanos Murga de Córdoba (GARCIA Y BELLIDO, 1961).

\section{Estancia VIII: (Fig. 4)}

Esta habitación, a la que se accede directamente desde el peristilo, comunica además con el espacio LVIII, formando ambos lo que Carrillo define como una simplificación del esquema alcoba-antecámara sin recurrir, como en el caso de las estancias 
LIX, LX y LXII, a un distribuidor (CARRILLO, 1992, 296). En tal esquema, para cuya interpretación Carrillo vuelve a apoyarse en los estudios de Thebert sobre las casas norteafricanas (THEBERT, 1988, 349-350), la estancia VIII sería antecámara, con cierto valor incluso de representación, sirviendo en definitiva de vestíbulo y paso a la estancia LVIII, totalmente interior y de menos pretensiones decorativas, como demuestra su pavimento de cal.

El esquema compositivo del mosaico que ocupa la estancia VIII (HIDALGO, 1991, $\mathrm{N}^{\circ} 3,333$ ss.; Láms. V-VI) es el de un cuadrado cuyo centro lo forma un octógono desarrollado, con un círculo inscrito en el que se inserta a su vez otro cuadrado tangente conformado mediante bandas de damero polícromo, dejando entrever una línea de cheurones superpuestos polícromos (BALMELLE, 1985, 35, pl. 8,b) rodeada de otra con postas de enrollado sencillo en círculo (BALMELLE, 1985, 156, pl.101, b.); el octógono se rodea todo él de figuras geométricas simples, cuadrados, de interiores decorados con los siguientes esquemas geométricos:

- marco formado por una trenza doble polícroma con un cuadrado dentellado inscrito. Es utilizado en 4 de los cuadrados, afrontados dos a dos y alternados con el resto;

- cuadrado en el que se inscriben dos peltas afrontadas, todo él rodeado de espinas rectilíneas cortas (BALMELLE, 1985, 40, pl. 11, d.)

- cuadrado con el mismo motivo interno, pero rodeado de dientes de sierra con borde escalonado.

Entre los cuadrados se desarrolla un esquema de triángulos, unos bícromos y otros con hojas de yedra incritas, alternando unos con otros.

En los ángulos del mosaico se sitúan cuartos de círculo enmarcados por tres filetes, uno simple, otro formado por triángulos y el siguiente por dientes de sierra; en su interior un cuadrado en el que se inscribe otro tangente, cóncavo y bícromo.

En el acceso a la estancia VIII desde el peristilo, el mosaico incorpora un damero bícromo, en blanco y negro, que ocupa el espacio dejado por la puerta.

En cuanto a los paralelos de este pavimento no creemos necesario señalar más de los ya aportados por el primer investigador que los estudió (HIDALGO, 1991).

Estancia XIX: (HIDALGO, 1991, Nº 5, 337 ss.; Láms. IX-XIII) (Fig. 5)

Esta estancia, asociada a la XXXIII que la precede, es considerada por Carrillo como "polivalente, sin función definida y sin una relación directa entre forma y función" (CARRILLO, 1992, 295), perteneciendo, eso sí, al espacio privado de la villa. Carrillo descarta su identificación como dormitorio, basándose para ello en que los vanos de entrada de las estancias XXXIII y XIX quedan situados en el mismo eje, por lo que la luz, en el caso de que se tratase de un cubiculum, incidiría directamente desde el peristilo a la estancia XIX (CARRILLO, 1992, 297; Cfr. PACKER, 1967, 136, $\left.n^{\circ} .31\right)$. 
Para el citado investigador la funcionalidad de este espacio pudo ser la de servir como biblioteca, comedor familiar o sala de descanso (CARRILLO, 1992, 297); sin embargo, hemos de matizar esta opinión, inclinándonos a pensar, de acuerdo con la opinión emitida recientemente por Vaquerizo, con base en la reconstrucción ideal por ordenador del pavimento de esta estancia, que se trata de un cubiculum dormitorium (VAQUERIZO et alii, 1994, 112), ya que la composición geométrica del mosaico utiliza un esquema en el que se establece una división decorativa entre el espacio reservado para el lecho, en el extremo sur, y el resto de la estancia, siendo el primero de composición más sencilla que el segundo.

Los lados menores del mosaico aparecen decorados con sendas franjas de roleos vegetales en negro.

Por su parte, el espacio de mayor amplitud queda enmarcado por una franja de dientes de sierra dentellados, paralela una trenza doble polícroma, dando lugar a un cuadrado en el que se insertan dos más superpuestos hasta conformar un estrella de ocho puntas cuyo centro lo ocupa un círculo ancho de guiloches con el centro recto y trazado (BALMELLE, 1985, 124, pl.74, f.), que nos ha llegado muy fragmentado.

Esta mala conservación afecta de manera especial al extremo meridional del mosaico, donde presumiblemente debió colocarse el lecho -si hemos de juzgar por el diseño del pavimento, así como por aquellos otros aspectos ya destacados más arriba-. Tal sector aparece enmarcado por un filete doble de teselas negras asociado a un filete de medias crucecitas no contiguas (BALMELLE, 1985, 170, pl. 113, C), que acoge a su vez el esquema decorativo diferenciado: un cuadrilóbulo de peltas -que a su vez enmarcan toda la composición- rodea a un cuadrado (BALMELLE, 1985, 356, pl. 228, c) delimitado por una línea denticulada y que acoge en su interior un motivo de aspas de molino tangentes (BALMELLE et alii, 1985, 48; Pl. 17, d). Le sigue un motivo, posiblemente circular, mediante varias líneas concéntricas, y, a nuestro juicio, según el esquema que hemos reconstruido mediante ordenador, se remataría la composición con otro cuadrado similar al primero, de forma muy semejante a como se observa en el mosaico de Fordongianus (ANGIOLILLO, 1987, 162-165, fig. 112) en Cerdeña, de fines del siglo II o comienzos del III, en el que entre los cuatrilóbulos de peltas se insertan círculos, si bien en el caso de El Ruedo tal motivo circular parece verse sustituido por otro un tanto indeterminado y de tendencia elipsoidal (Fig. 6).

Del mismo modo, podemos señalar algunos paralelos significativos en Tharros, donde en un mosaico de mediados del siglo III encontramos las mismas hojas fusiformes y nudos de Salomón inscritos en los cuadrados (ANGIOLILLO, 1981, nº. 125, pp.138139, fig. 33, lám. XXI), y en Ostia, donde también encontramos un motivo similar, fechado en la segunda mitad del siglo III en un mosaico bícromo (BECATTI, 1961, $n^{\circ} .400$, p. 210, lám. XLIII). Finalmente, son muchos los ejemplos del mismo tipo documentados -en su mayoría ya destacados por Hidalgo (1991)- en los pavimentos musivos del Norte de Africa; es el caso de la estancia 14 de la villa Gara de las Nereidas 
presso Taigiura, donde observamos el nudo de Salomón inscrito en un cuadrado así como la flor cuatripétala en un círculo (DE VITA, 1966, pp. 37-38, lám. IX a).

\section{CONCLUSION}

Ofrecemos en este artículo una brevísima aportación al conocimiento de los pavimentos musivos en la villa romana de El Ruedo, en el marco de un amplio Proyecto de Investigación que aún habrá de producir novedades importantes y que, a los efectos que a nosotros interesan, quedará en buena parte completo cuando finalice el proceso de reconstrucción informática del yacimiento. Tal circunstancia nos permitirá recrear los esquemas de casi todos sus mosaicos, así como una anastilosis gráfica de la villa que facilitará la visión de conjunto, apreciando con toda exactitud las distintas refectiones documentadas en el asentamiento; los sistemas decorativos -musivos, parietales, arquitectónicos o escultóricos- que se suceden ornamentando sus salas, y finalmente las cubiertas, la disposición rítmica de los distintos módulos que componen la villa, su posición en el paisaje y su exacto esquema arquitectónico, etc.

Son aspectos todos ellos sobre los que actualmente se trabaja y que, como pretendemos para el resto de Baetica -aún cuando somos conscientes de lo enormemente limitados que son los datos de que disponemos-, nos permitirá entender la decoración musivaria aplicada a complejos arquitectónicos públicos o privados desde una correcta valoración de todo lo relacionado con su técnica, su estilo, su procedencia artesanal, su iconografía y consiguiente valor simbólico, y, por supuesto, la funcionalidad de los establecimientos y estancias en que se utilizan, por cuanto ni todo el mundo podía, ni se utilizaban los mosaicos para todos los espacios de la casa.

Prima en la elección de los temas la oferta de cartones de que dispusieran los talleres más cercanos y en la disposición de los mosaicos el componente de privacidad que rige en las diversas estancias. Sin embargo, tanto en uno como en otro tema incidirían enormemente factores tales como la capacidad adquisitiva del comitente, su formación cultural y, sobre todo, el valor de representación y aparato que quisiera dar a las estancias que habían de recibir la ornamentación musivaria.

Aspectos que en buena medida exceden el análisis puramente formal y a cuya resolución queremos dedicar nuestros próximos esfuerzos. 


\section{Bibliografía}

AAVV (1989), Mosaicos Romanos. In Memoriam Nanuel Fernández-Galiano, Madrid, 1989.

AAVV (1990), Mosaicos Romanos. Estudios sobre iconografía. In Memoriam Alberto Balil, Guadalajara.

ANGIOLILLO, S. (1981), Sardinia: mosaichi antici in Italia. Roma.

_ (1987), L'Arte della Sardegna Romana, Milano.

ARCE, J. (1993), "Los mosaicos como documentos para la

historia de la Hispania tardía (siglos IV-V)", AEspA 66, Madrid, pp. 265-274.

BALMELLE, C. et alii (1985), Le décor géometrique de la mosaique romaine, Paris.

BECATTI, G. (1961). Mosaici e pavimenti marmorei. Scavi di Ostia, $M$.

BEDOJERE, G. de la (1993), Roman villas and the Countryside, English Heritage.

BLANCO, A., GARCIA, J., BENDALA, M. (1972), "Excavaciones en Cabra (Córdoba). La Casa del Mitra (Primera Campaña, 1972)". Habis 3, Universidad de Sevilla, pp. 297-319.

BLAZQUEZ MARTINEZ, J. M. (1993), Mosaicos Romanos en España, Madrid.

BLAZQUEZ MARTINEZ, J. M.; MEZQUIRIZ, M. A. (1985), Mosaicos romanos de Navarra, Corpus de España VII, Madrid.

CARRILlO, J. R. (1990), "Técnicas constructivas en la villa romana de El Ruedo (Almedinilla, Córdoba)", $A A C$ I, Córdoba, pp. 81-109.

-_ (1992), Análisis arquitectónico de la villa romana de El Ruedo (Almedinilla, Córdoba), Tesis Doctoral en 3 vols. (inédita), Univ. de Córdoba.

DAVIAULT, A., LANCHA, J., LOPEZ PALOMO, A., (1987), "Un mosaico con inscripciones/Une mosaique á inscripitions, Puente Genil (Córdoba). Publications de la Casa de Velázquez. Etudes et Documents, fasc. III, Madrid.

DURAN, M. (1993), Iconografía de los mosaicos romanos en la Hispania Altoimperial, Barcelona.

ENNABLI BEN OSMAN, W. (1983), "La maison de la Valiere a Carthage. L'architecture", en Mosaique. Recueil d'hommages a Henri Stern, Paris, pp. 129-145.

FERNANDEZ CASTRO, M. C. (1978), “Aspectos arquitectónicos musivarios de las villas romanas en Andalucía”, Actas I Congreso de Historia de Andalucía. Fuentes y Metodología. Andalucía en la Antigüedad, Córdoba, pp. 309-331.

- (1982), Villas romanas en Hispania, Madrid.

FERNANDEZ GALIANO, D. (1980), Mosaicos romanos de esquema a compás, Guadalajara.

- (1987), Mosaicos Romanos del Convento Caesaraugustano, Zaragoza.

— (1989), "La villa de Materno", Mosaicos Romanos. In Memoriam Manuel Fernández-Galiano, Madrid, pp. 255-270.

GARCIA Y BELLIDO, A. (1961), "Parerga de Arqueología y Epigrafía hispano-romanas", B.R.A.C. 82, Córdoba, pp. 384 ss.

GOMEZ PALLARES, J. (1992), "Reflexión sobre un 'corpus' de inscripciones sobre mosaico en Hispania", Faventia 14-2, pp. 33-53.

— (1993), "Inscripciones musivas en la antigüedad tardía hispana", AEspA, 66, 1993, pp. 285-293.

GUARDIA PONS, M. (1992), Los mosaicos de la Antigüedad Tardía en Hispania, Barcelona.

HIDALGO PRIETO, R. (1991), "Mosaicos con decoración geométrica y vegetal de la villa romana de El Ruedo (Almedinilla, Córdoba)", AAC 2, Univ. de Córdoba, pp. 325-362.

_ (1994), "Mosaicos de la villa romana de El Ruedo (Almedinilia, Córdoba)", VI Coloquio Internacional sobre el Mosaico Antiguo. Palencia-Mérida. 1990, Guadalajara, pp. 15-22. 
JARREGA DOMINGUEZ, R. (1993), "EI mosaico polícromo con decoración geométrica de círculos intersecantes de la villa romana de Barrugat (Bitem, Tarragona)", AEspA 66, Madrid, pp. 275-283.

JIMENEZ SALVADOR, J. L.; MARTIN-BUENO, M. A. (1992), La Casa del Mitra. Cabra, Córdoba, Cabra.

LOPEZ MONTEAGUDO, G. (1991), "La caza en el mosaico romano". Iconografía y simbolismo. Arte, sociedad, economía y religión. Homenaje a J.M. Blázquez, pp. 497-512.

MORERE, N. (1989), Las villae romanas en la Galia Narbonense, Madrid.

NOLLA, J. M.; SAGRERA, J. (1993), "Els mosaics de la villa romana del Pla de l'Horta (Sarriá de Ter)", Cypsela X, Girona, pp. 145-158.

OVADIAH, A. (1980), Geometrics and Florals Patterns in Ancient Mosaics, Roma.

PACKER, J. E. (1967), "The Domus of Cupid and Psyche in ancient Ostia” AJA 71, pp. 123-131.

RAMALlo ASENSIO, S. (1985), Mosaicos romanos de Carthago Nova (Hispania Citerior), Murcia.

REBUFFAT, R. (1969), "Maisons a peristyle d'Afrique du Nord. Répertoire de plans publiés", MEFRA LXXXI.2, pp. 657-724.

RODRIGUEZ OLIVA, P. (1987), Mosaicos romanos de Bobadilla (Málaga), Málaga.

SALIERS, G. (1974), "Untersuchungen zu den geometrischen Gliederugsschemata romischer Mosaiken", B.J., CLXXIV, pp. 1-178.

SIERRA FERNANDEZ, J. A. de la (1994), "Sigillatas africanas y otras cerámicas de mesa tardías de la villa de El Ruedo", $A A C$ 5, Univ. de Córdoba (e.p.).

SMITH, D. J. (1963), "Three Fourth-Century of mosaics in Roman Britain", Colloques Internationaux du Centre national de la Recherche Scientifique. La Mosaique Greco-Romaine, Paris, pp. 95-116.

— (1975), "Roman Mosaics in Britain before the fourth century", La mosaique Greco-Romaine, II, Paris, pp. 269 ss.

THEBERT, Y. (1988), "Vida privada y arquitectura doméstica en el Africa romana", en ARIES, Ph.; DUBY, G. (Dirs.), Historia de la vida privada. I. Del Imperio Romano al año mil, Madrid, pp. 304-401.

VAQUERIZO, D. (1990a), "La villa romana de El Ruedo (Almedinilla, Córdoba)", AEspA 63, Madrid, pp. 295-316.

_- (1990b), "La decoración escultórica de la villa romana de El Ruedo (Almedinilla, Córdoba)", AAC I, Univ. de Córdoba, pp. 125-155.

VAQUERIZO, D.; CARRILLO, J. R. (1994), “La villa romana de El Ruedo (Almedinilla, Córdoba)”, JRA, University of Michigan (e. p.).

VAQUERIZO, D. et alii (1994), Arqueología Cordobesa. Almedinilla, Córdoba.

VIEGAS, C., ABRA OS, F., MACEDO, M. (1993), Dicionário de motivos geométricos no mosaico romano.

VITA, A. di (1986) La villa della "Gara della Nereide" presso Tagiura: un contributo alla storia del mosaico romano. Trípoli.

WARD-PERKINS, J. B. (1981), Roman Imperial Architecture, Harmands Worth. 

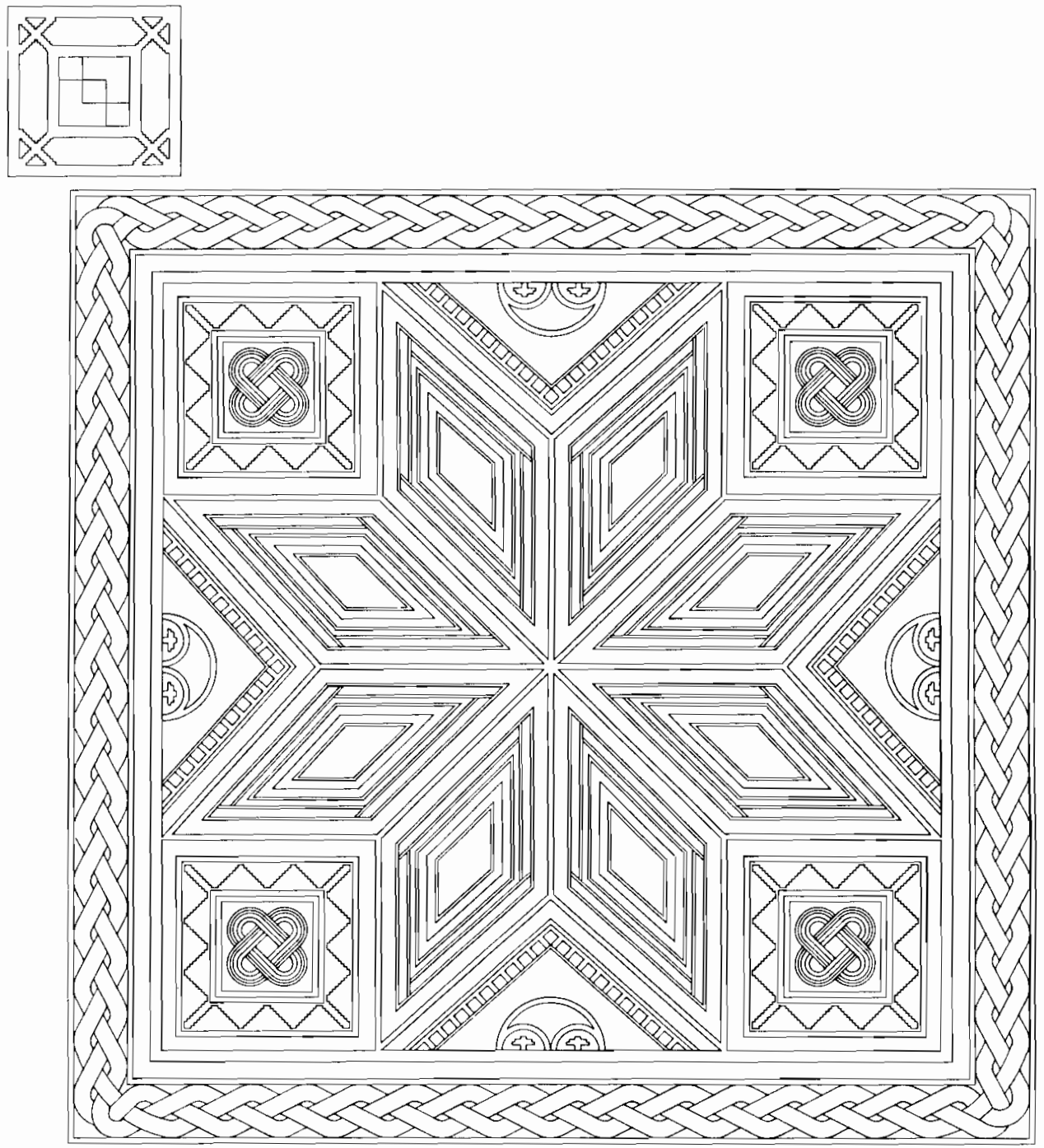

Fig. 1: Esquema ideal informatizado del pavimento musivo documentado en la estancia LIX (según J. L. Vaquerizo). 


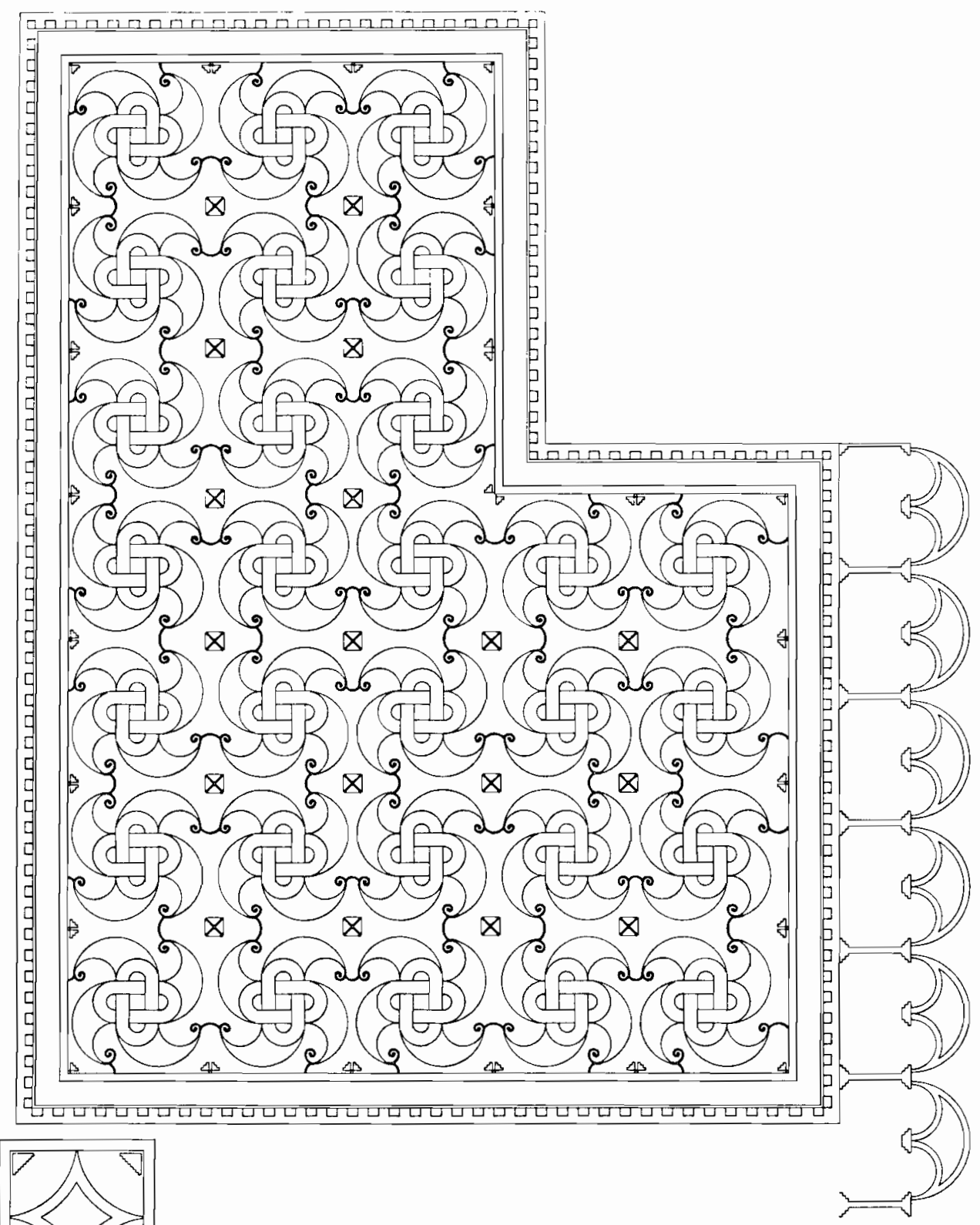

Fig. 2: Esquema ideal informatizado de pavimento musivo documentado en la estancia LXI (según J. L. Vaquerizo). 


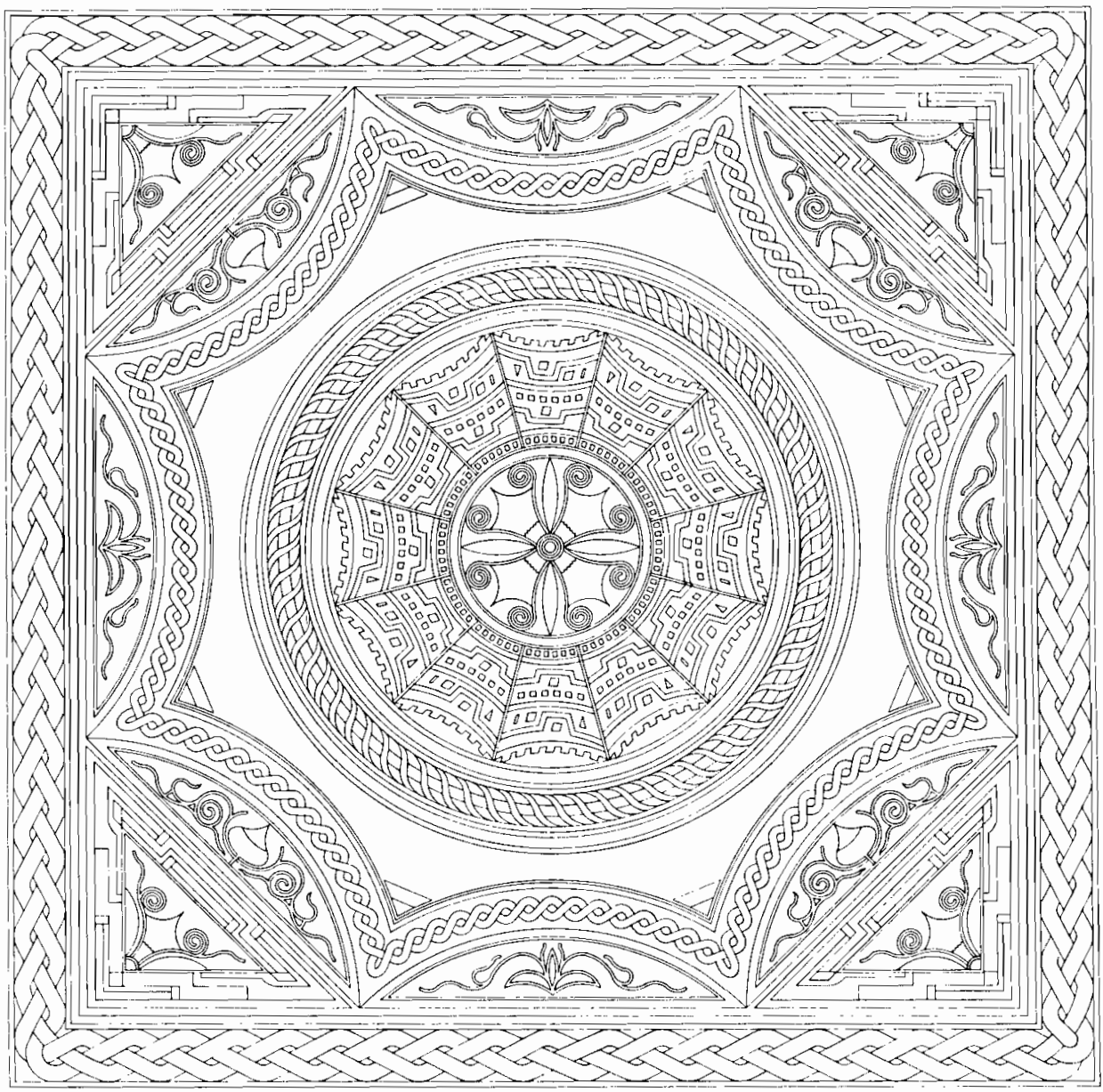

Fig. 3: Esquema ideal informatizado del pavimento musivo documentado en la estancia XXXVII (según J. L. Vaquerizo). 


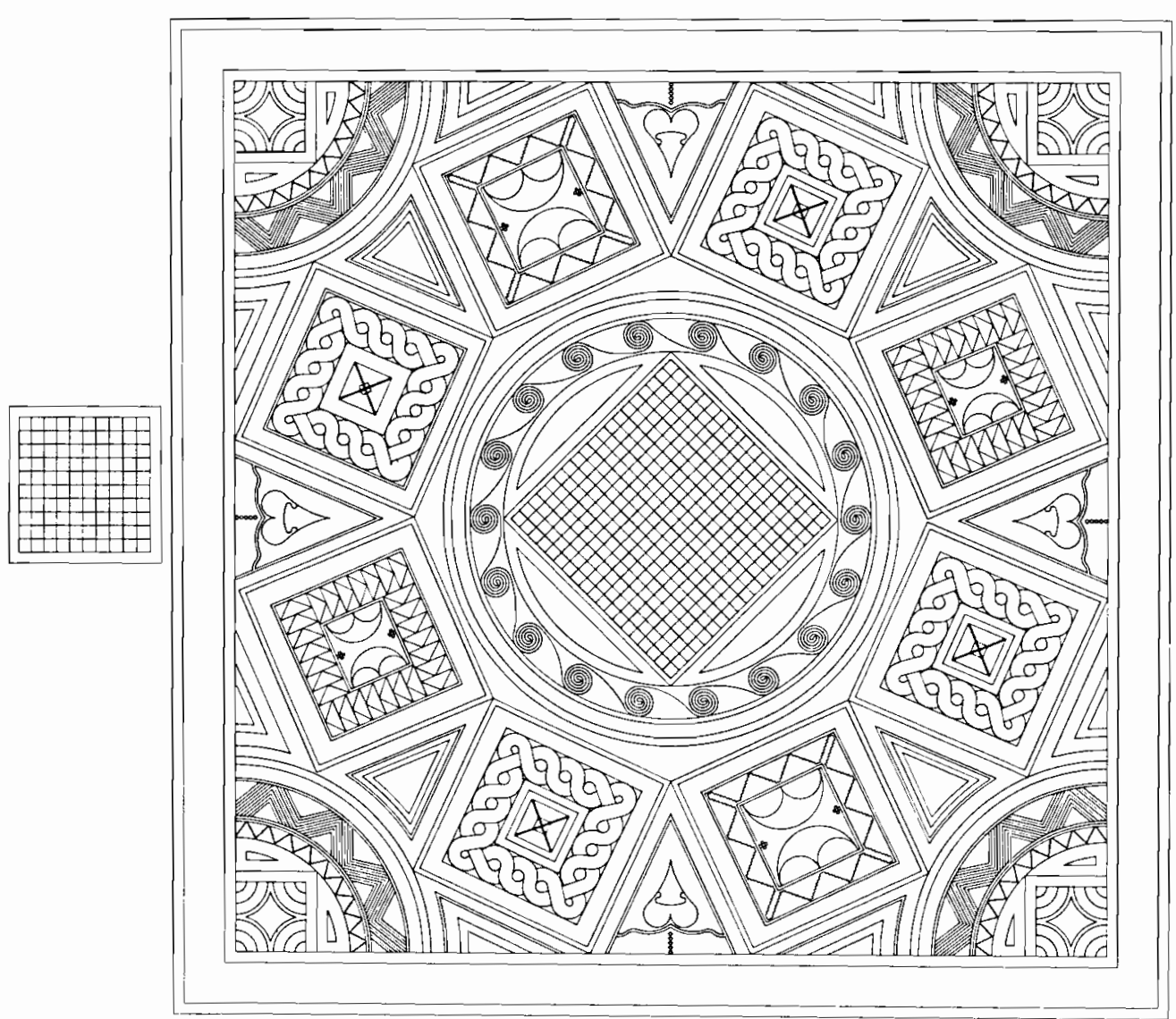

Fig. 4: Esquema ideal informatizado del pavimento musivo documentado en la estancia VIII (según J. L. Vaquerizo). 


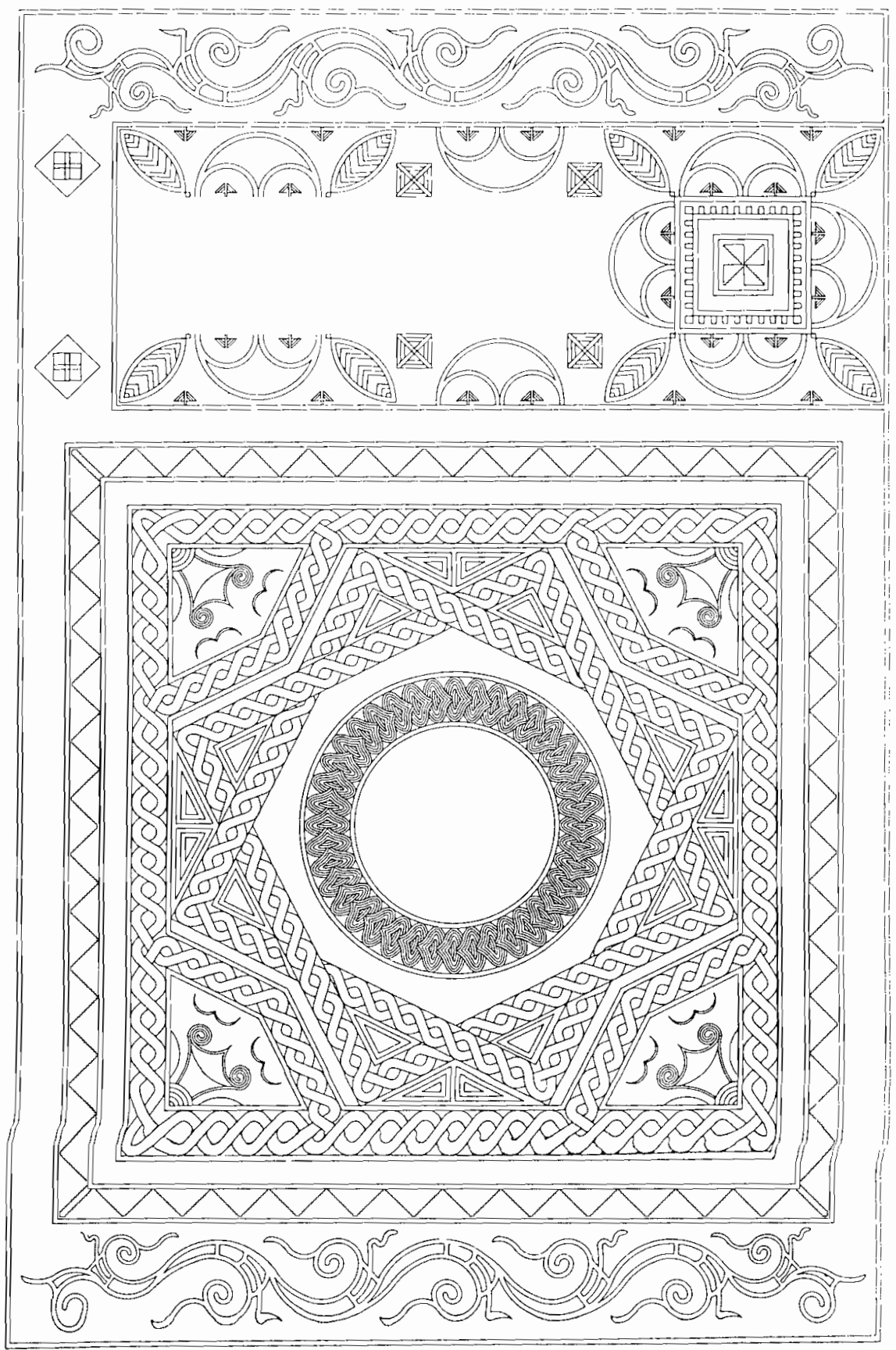

Fig. 5: Esquema ideal informatizado del pavimento musivo documentado en la estancia XIX, a partir exclusivamente de los restos que nos han llegado (según J. L. Vaquerizo). 


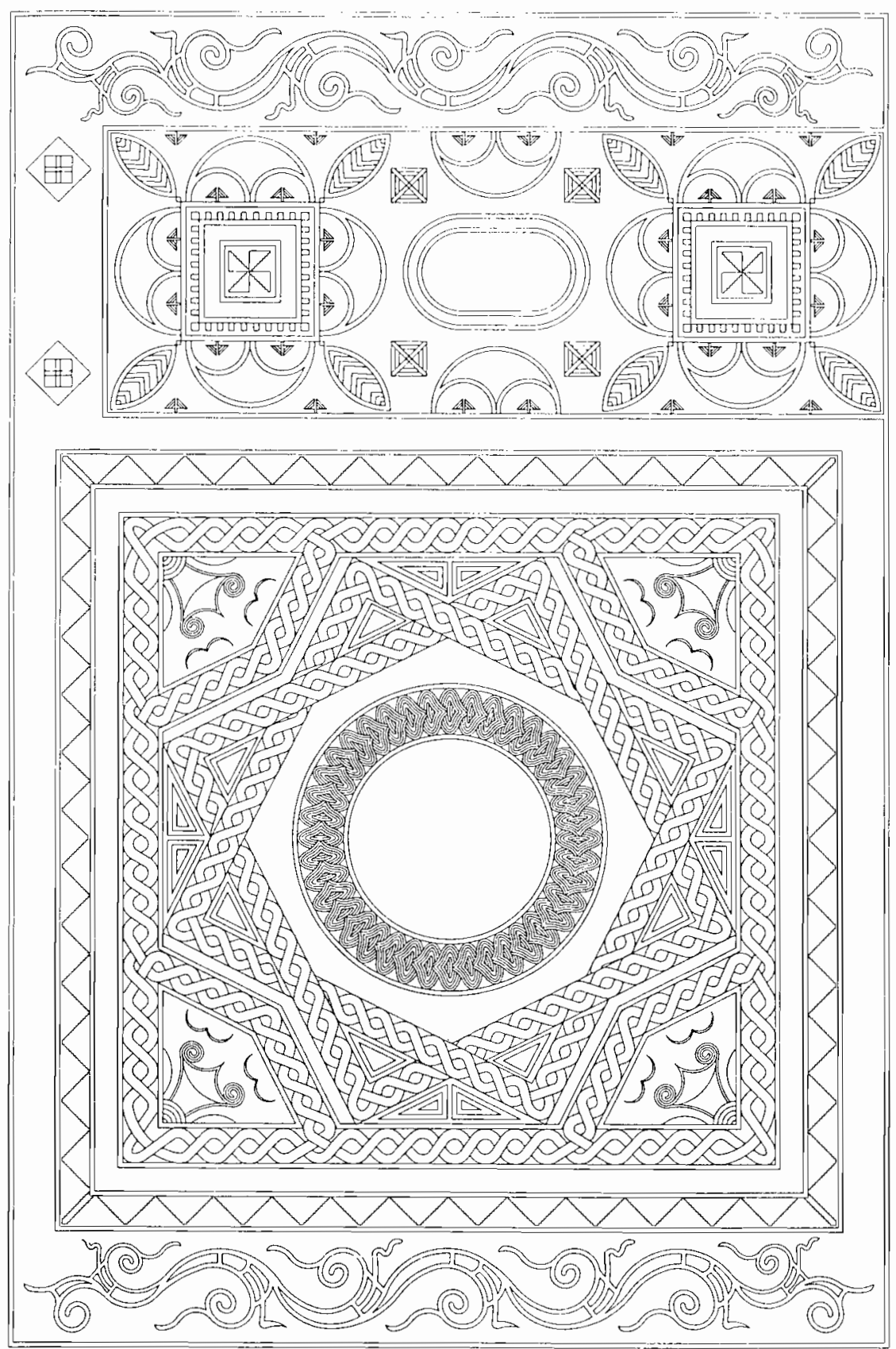

Fig. 6: Esquema ideal informatizado del mosaico recogido en la Fig. 5, con la reconstrucción ideal del esquema geométrico que, a nuestro juicio, ocuparía el extremo septentrional del la estancia (según J. L. Vaquerizo). 